\title{
Case report involving temporomandibular dysfunction, eagle's syndrome and torus mandibularis -Multidisciplinary approaches
}

\author{
Takami Hirono Hotta ${ }^{1}$, Cássio Edvard Sverzut ${ }^{2}$, Marcelo Palinkas ${ }^{3 *}$, César Bataglion ${ }^{3}$, \\ Melissa Oliveira Melchior ${ }^{3}$, Patrícia Tiemy Hirono Hotta ${ }^{4}$, Sérgio Olavo Petenusci ${ }^{5}$, \\ Simone Cecilio Hallak Regalo ${ }^{5}$ \\ ${ }^{1}$ Department of Dental Materials and Prosthodontics, Ribeirão Preto School of Dentistry, University of São Paulo, Ribeirão Preto,
Brazil
${ }^{2}$ Department of Oral and Maxillofacial Surgery and Periodontology, Ribeirão Preto School of Dentistry, University of São Paulo,
Ribeirão Preto, Brazil
${ }^{3}$ Department of Restorative Dentistry, Ribeirão Preto School of Dentistry, University of São Paulo, Ribeirão Preto, Brazil
${ }^{4}$ Department of Surgery, Prosthodontics and Maxillofacial Traumatology, São Paulo Dental School, University of São Paulo, São
Paulo, Brazil
${ }^{5}$ Department of Morphology, Stomatology and Basic Pathology, Ribeirão Preto School of Dentistry, University of São Paulo,
Ribeirão Preto, Brazil \\ Email: "palinkas@usp.br
}

Received 6 August 2013; revised 6 September 2013; accepted 23 September 2013

Copyright (C) 2013 Takami Hirono Hotta et al. This is an open access article distributed under the Creative Commons Attribution License, which permits unrestricted use, distribution, and reproduction in any medium, provided the original work is properly cited.

\begin{abstract}
The prevalence of torus mandibularis, Eagle's syndrome and parafunctional activity was reported to be higher in patients with temporomandibular joint dysfunction and might be useful as an indicator of increased risk of temporomandibular disorders. This study case report was to evaluate a 62-year-old female patient, with limited mandibular and cervical movements, articular and muscular pain, ear fullness, irritation of the lingual mucosa, discomfort associated with movement of her tongue, involving temporomandibular dysfunction, torus mandibularis and Eagle's syndrome. The treatment comprehended the collection of data of Helkimo's Clinical Dysfunction Index, electromyographic activity of the masseter and temporalis muscles in rest position and bite force, masticatory efficiency, speech evaluation and oclusal splint, after the excision of the stylohyoid process and mandibularis torus. The authors found a decrease in electromyographic activity of masticatory muscles, a reduction of painful symptoms throughout the region muscular and joint pains, an increase in the amplitude of mandibular movements, an improvement in masticatory efficiency and an increase in bite force.
\end{abstract}

Keywords: Temporomandibular Dysfunction; Eagle's

${ }^{*}$ Corresponding author.
Syndrome; Torus Mandibularis; Electromyography; Bite Force

\section{INTRODUCTION}

Torus mandibularis (TM) is an exophytic bone growth that usually occurs bilaterally along the lingual surface of the premolar area of the mandible, superior to the mylohyoid ridge $[1,2]$. In patients who are completely or partially edentulous, the occurrence of TM can interfere with the prosthetic treatment plan in dentate patients. TM can interfere with masticatory and speech functions. Therefore, even when asymptomatic, the surgical removal of the tori is indicated in these cases $[3,4]$.

The etiology of TM has been investigated by several authors; however, no consensus has been reached. It is generally accepted that genetic factors contribute to the occurrence of TM [5-8].

The prevalence of both TM and parafunctional activity has been reported to be higher in patients with temporomandibular joint dysfunction [9], and therefore, these factors may be useful indicators of an increased risk of temporomandibular disorders in some patients.

Eagle's syndrome affects the head and neck structures and produces headache, earache, changes in swallowing and speech, and facial, cervical and eye pain [10-14].

Symptoms may also occur along the distribution of the external and internal carotid artery branches due to com- 
pression at points on the vessels, affecting vascular circulation and irritating the sympathetic nerves of the arterial sheath [15].

It is often difficult to establish a definitive diagnosis of Eagle's syndrome due to the similarity of these symptoms to the other pathologies that affect the head and neck areas. Therefore, the use of an occlusal splint, normally applied in the treatment of TMD, can be helpful during the process of differential diagnosis [16].

Eagle's syndrome affects approximately $27 \%$ of the population, and elderly females show a greater prevalence of an elongated stylohyoid ligament complex $[18,19]$. Additionally, the presence of an elongated stylohyoid ligament complex was found in approximately $19.6 \%$ of Brazilian human skulls [17]. This syndrome is more prevalent in the elderly because the aging process promotes the development of a tendinosis at the junction of the stylohyoid ligament and the lesser horn of the hyoid bone [20].

The underlying mechanisms for this life-long process may involve variability in the length and shape of the second branchial arch cartilage, which may explain cases of styloid complex ossification in adults and the elderly. It is also possible that the ossification of the branchial cartilages is a part of the normal aging process, as it also occurs in other derivatives of the branchial arches, such as the laryngeal cartilages [21].

The aims of this present study were to demonstrate different approaches for the resolution of a clinical case involving temporomandibular dysfunction, torus mandibularis and Eagle's syndrome in an elderly woman.

\section{CASE REPORT}

A 62-year-old woman was referred to the Occlusion, Temporomandibular Dysfunction and Orofacial Pain in Patients with Especial Care Course, School of Dentistry of Ribeirão Preto, University of São Paulo, complaining of limited mandibular and cervical movements, articular and muscular pain, ear fullness, irritation of the lingual mucosa, and discomfort associated with movement of her tongue. Earlier, she received a medical diagnosis of Eagle's syndrome, and surgical removal of the styloid process was suggested.

The patient was informed about the treatment plan and study procedures by the investigators and signed the informed consent. The study was approved by the Ethics Committee of the School of Dentistry of Ribeirão Preto, University of São Paulo (process n 2006.1.971.58.5).

A physical examination of the oral cavity revealed general dental wear and extensive bony overgrowths along the lingual surface in the premolar area of the mandible (Figure 1). At the time of examination, the mucosa that was overlying the tori appeared normal; however, during later preoperative appointments, small areas of abrasive lesions were often observed. Furthermore, the tongue rest position and the functional activities of the masticatory system were abnormal, promoting muscular pain. The patient revealed that this pain often occurred after the mastication of hard food; she also reported occasional bruxism.

The first stage of the treatment plan involved the collection of relevant data with the following analyses: Helkimo index [22], electromyographic activity of the masseter and temporalis muscles in rest position (Table 1), bite force (Table 2) [23], masticatory efficiency [24] and speech evaluation [25].

The orofacial muscles (masseter, temporalis, pterygoid) and the condyle lateral pole were bilaterally palpated in order evaluate pain upon palpation using a numeric scale.

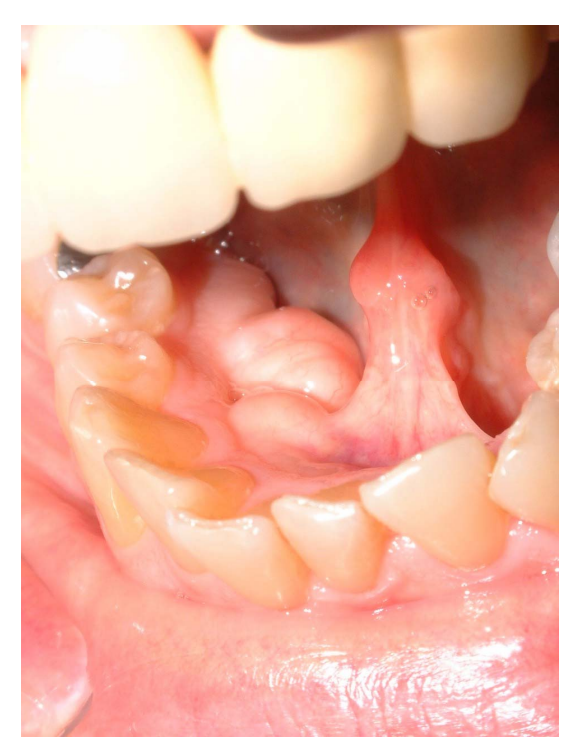

Figure 1. Extensive bony overgrowths in the premolar area of the mandible.

Table 1. Numeric values of the electromyographic activities of the masseter and temporalis muscles, before (E1) and after (E2) treatments.

\begin{tabular}{ccccc}
\hline \multicolumn{5}{c}{ Rest Position $(\mu \mathrm{V})$} \\
\hline Phases & $\begin{array}{c}\text { Right } \\
\text { Masseter }\end{array}$ & $\begin{array}{c}\text { Left } \\
\text { Masseter }\end{array}$ & $\begin{array}{c}\text { Right } \\
\text { Temporalis }\end{array}$ & $\begin{array}{c}\text { Left } \\
\text { Temporalis }\end{array}$ \\
\hline E1 & 10.5 & 4.8 & 4.3 & 4.6 \\
E2 & 6.1 & 2.7 & 4.3 & 4.1 \\
\hline
\end{tabular}

Table 2. Means of the maximal bite force in the left and right molar regions, before (E1) and after (E2) treatments.

\begin{tabular}{ccc}
\hline Site & Evaluation & Bite Force (N) \\
\hline \multirow{2}{*}{ Right Molar } & E1 & 34.3 \\
& E2 & 46 \\
Left Molar & E1 & 67.6 \\
& E2 & 88.2 \\
\hline
\end{tabular}


This scale consisted of 11 points, beginning with " 0 " (no pain) and ending in "10" (the worst imaginable pain) [26] (Table 3).

Using the Hugoson Index [27], the observed occlusal wear was classified as level 1 (attrition of enamel down to dentin spots), confirming the presence of bruxism that was reported earlier by the patient.

Maximum mouth opening and the right and left lateral movements were measured with a millimeter ruler. To measure the maximum mouth opening movement, the patient was requested to open her mouth, and the distance between the incisal borders was measured. The patient performed right and left lateral movements, and the distance between the mandibular and maxillary midlines was measured. As there was no coincidence, a pencil mark was made on the surface of the maxillary incisor, corresponding to the mandibular midline, and a measurement was later taken. The protrusion distance between the maxillary and mandibular incisal borders was measured with a ruler (Table 4).

The patient's masticatory efficiency was determined using a coconut and sieve system, before and after treatment. The patient received four aliquots of coconut pieces, weighing a total of $20 \mathrm{~g}$. Each piece of food was chewed for 50 cycles. After mastication, the chewed particles were expelled into a set of five sieves $(5,4,3,2$ and $1 \mathrm{~mm}$ ) and were washed with water. Then, the particles were separated according to the granulometric mesh particles contained in each sieve, and these particles were put inside test tubes (A, B, C, D and E), according their sizes. The test tubes were centrifuged for 5 minutes, and the volumetric data were inserted in a mathematical formula $(4 \mathrm{~A}+2 \mathrm{~B}+\mathrm{C} / \mathrm{D}+\mathrm{E})$ to obtain the index of mastication $(>10=$ optimal, 5.0 to $9.9=$ good, 2.0 to $4.9=$ regular, 1.0 to $1.9=$ bad, $<1.0=$ terrible) (Table 5).

Afterward, the TMs were completely removed under local anesthesia. The inferior alveolar nerves were

Table 3. Intensity of muscular and articular pain, before (E1) and after (E2) treatments.

\begin{tabular}{ccccccccc}
\hline & \multicolumn{2}{c}{ Masseter } & \multicolumn{2}{c}{ Temporalis } & \multicolumn{2}{c}{$\begin{array}{c}\text { Lateral } \\
\text { Pterygoid }\end{array}$} & \multicolumn{2}{c}{$\begin{array}{c}\text { Condyle } \\
\text { Lateral Pole }\end{array}$} \\
\cline { 2 - 9 } & Right & Left & Right & Left & Right & Left & Right & Left \\
\hline E1 & 7 & 5 & 8 & 3 & 8 & 7 & 8 & 2 \\
E2 & 7 & 2 & 2 & 0 & 3 & 0 & 5 & 0 \\
\hline
\end{tabular}

Table 4. Numeric values of the measurements of mandibular movements, before (E1) and after (E2) treatments.

\begin{tabular}{ccccc}
\hline \multicolumn{5}{c}{ Mandibular Movements (mm) } \\
\hline & $\begin{array}{c}\text { Mouth } \\
\text { Opening }\end{array}$ & $\begin{array}{c}\text { Right } \\
\text { Laterality }\end{array}$ & $\begin{array}{c}\text { Left } \\
\text { Laterality }\end{array}$ & Protrusion \\
\hline E1 & 29 & 5 & 4 & 2 \\
E2 & 50 & 8 & 9 & 3 \\
\hline
\end{tabular}

blocked with $1.8 \mathrm{ml}$ of anesthetic solution containing mepivacaine (2 percent) and epinephrine (1:100.000), applied to each side. The surgical approach consisted of an intrasulcular incision that extended from one first molar to the other. Afterward, a full-thickness flap was carefully elevated, exposing both TMs and preserving the insertion of the genioglossus muscles. The delimitation of the TMs was performed using a surgical length \#702 taper fissure crosscut carbide bur. Afterward, a thin, straight chisel was positioned into the gap and lightly hammered twice, resulting in the easy removal of the TMs. The bone irregularities were removed with a manual Seldin bone file \#12 to obtain a smooth surface along the lingual cortical. The surgical field was copiously irrigated with sterile saline solution, and the wound was sutured in a non-continuous fashion with 4 - 0 black silk thread mounted in a $1.7 \mathrm{~cm}-1 / 2$ circle needle (Ethicon, São Paulo, Brazil). The biopsied tissues were sent to the Department of Morphology, Stomatology and Physiology for histopathological analysis, which confirmed the diagnosis of TM. The patient was medicated with sodic diclofenac (50 mg every 8 hours for 3 days) and dipiron (500 mg every 8 hours, to prevent pain). The postoperative period was uneventful, and the final result was a success.

After 2 months, an acrylic splint with anterior guidance for disocclusion in protrusion and canine guidance for disocclusion in lateral movements was provided to the patient, who wore it for 6 weeks and was submitted to weekly follow-up.

\section{RESULTS}

The patient exhibited intense signs and symptoms of dysfunctions, such as limited jaw opening, pain during mandibular movements, and pain during articular and muscular palpation. She received an Acute Physiology and Chronic Health Evaluation III score (AIII) for anamnestic dysfunction and, after treatment, for minor signs and symptoms of dysfunction, such as TMJ noises, fatigue of masticatory muscles and TMJ stiffness.

The data collected on the evaluation of mandibular movements, functional evaluation of the TMJ, pain during mandible movements, muscular pain and articular pain accurately demonstrated the degree of TMJ dysfunction, both before (DiIII-severe clinical symptoms

Table 5. Numeric values of the volumetric data and masticatory index, before (E1) and after (E2) treatments.

\begin{tabular}{cccccccc}
\hline \multicolumn{7}{c}{ Masticatory Efficiency } \\
\hline & Sieve & Sieve & Sieve & Sieve & Sieve & Results & Index \\
& A & B & C & D & E & Rad \\
\hline E1 & 2 & 4 & 10 & 10 & 4 & 1.85 & bad \\
E2 & 3 & 6 & 10 & 5 & 1 & 5.66 & good \\
\hline
\end{tabular}


of dysfunction) and after (DiII—moderate clinical symptoms of dysfunction) treatments.

\section{DISCUSSION}

Orofacial pain, joint noise and limited mandibular movements are symptoms commonly associated with TMD. Other symptoms, such as headache, earache, neck pain, tinnitus and dysphagia are also related to this disorder [28-30]. Similar signs and symptoms may be found in other diseases, such as Eagle's syndrome, and it may be difficult to establish the correct diagnosis and appropriate treatment $[10,31]$.

To facilitate diagnosis, the patient should be submitted to a group of professionals from diverse disciplines, to provide a comprehensive assessment and consultation in cases that present several signs and symptoms, such as the case reported here.

The first stage of the treatment plan involved the collection of data that allowed a global view of the patient's clinical and functional conditions. The sequence of procedures was then selected.

The surgical treatment for Eagle's syndrome involves the excision of the stylohyoid process $[10,12]$. However, such treatment involves the possibility of associating pathologies that may aggravate the symptoms. Therefore, a conservative, nonsurgical approach with an occlusal splint is often.

Dentists should be aware of the risks of surgical intervention to provide a better diagnosis and therapeutic procedure [32].

Because the occurrence of bilateral TM can interfere with masticatory and speech functions in dentate patients, affecting their quality of life, the clinicians in this case chose to apply surgical intervention. The favorable clinical results (tongue rest position and functional activeties) confirmed the appropriateness of this choice.

The ability to grind food is the end product of chewing, and several structures of the stomatognathic system work together so that chewing can be satisfactorily performed. To evaluate the masticatory function, which encompasses the efficiency of the masticatory muscles and masticatory performance, the maximum bite force is useful in both demonstrating how the system is fully interfaced and in revealing any anatomical changes and temporomandibular disorders that lead to an imbalance of this system [33].

After treatment, the patient's masticatory efficiency improved. The activity began to be pleasant, without joint or muscular pain and without trauma to the area that previously accommodated the mandibular tori. The patient could also chew food harder and faster.

Occlusal splint therapy was applied to treat the patient's TMD and to assist the group of professionals during the differential diagnosis process, as previously sug- gested [16]. The satisfactory clinical outcomes were evidenced in the increased amplitude of mandibular movements, decrease in muscular/articular pain and the improvement in functional activities.

The patient was instructed to chew pieces of test food as usual, and it was observed that chewing was performed on the left, with greater bite force on the left side. The right side exhibited greater muscle and joint pain, justifying the patient's preference for chewing on the left side. It should be noted that the magnitude of the bite force varies when characteristics of the craniofacial complex are abnormal, especially in cases of temporomandibular dysfunction [34].

Electromyographical analysis of the masseter and temporalis muscles may be used to compare muscular activities during rest and during clinical manipulation [18]. The results of electromyographic analyses showed that mean muscle activity was improved after the combination of surgical therapy and the occlusal splint.

This article reports a clinical case in which a combination of surgical therapy and dental resources were used to treat limited mandibular movements and painful symptoms in a patient with temporomandibular dysfunction, Eagle's syndrome and torus mandibularis.

In recent decades, the required care for older people has increased in the daily practice of dental professionals. Therefore, it is critical for clinicians to understand the situations that lead to discomfort in older patients.

\section{CONCLUSION}

In summary, dental practitioners should understand the causes of anatomical changes, improving their patients' quality of life by providing a more practical approach to the treatment of painful sintomatoligia in older people with Eagle's syndrome, temporomandibular disorder and mandibular tori.

\section{ACKNOWLEDGEMENTS}

The authors wish to thank Ribeirão Preto School of Dentistry, University of São Paulo.

\section{REFERENCES}

[1] Antoniades, D.Z., Belazi, M. and Panayiotis, P. (1998) Concurrence of torus palatinus with palatal and buccal exostoses-Case report and review of the literature. Oral Surgery, Oral Medicine, Oral Pathology, Oral Radiology, and Endodontology, 85, 552-557. http://dx.doi.org/10.1016/S1079-2104(98)90290-6

[2] Hassan, K.S., Alagl, A.S. and Abdel-Hady, A. (2012) Torus mandibularis bone chips combined with platelet rich plasma gel for treatment of intrabony osseous defects: Clinical and radiographic evaluation. International Journal of Oral and Maxillofacial Surgery, 41, 1519-1526. 
http://dx.doi.org/10.1016/j.ijom.2012.02.009

[3] Al-Bayaty, H.F., Murti, P.R., Matthews, R. and Gupta, P.C. (2001) An epidemiological study of tori among 667 dental outpatients in Trinidad \& Tobago, West Indies. Inte national Journal of Dentistry, 51, 300-304. http://dx.doi.org/10.1002/j.1875-595X.2001.tb00842.x

[4] Raldi, F.V., Nascimento, R.D., Sá-Lima, J.R., Tsuda, C.A. and Moraes, M.B. (2008) Excision of an atypical case of palatal bone exostosis: A case report. Journal of Oral Science, 50, 229-331.

http://dx.doi.org/10.2334/josnusd.50.229

[5] Matheus, R.A., Ramos-Perez, F.M., Menezes, A.V., Ambrosano, G.M., Haiter-Neto, F., Bóscolo, F.N. and de Almeida, S.M. (2009) The relationship between temporomandibular dysfunction and head and cervical posture. Journal of Applied Oral Science, 17, 204-208. http://dx.doi.org/10.1590/S1678-77572009000300014

[6] Eggen, S. and Natvig, B. (1991) Variation in torus mandibularis prevalence in Norway: a-statistical analysis using logistic regression. Community Dentistry Oral Epidemiology, 19, 32-35. http://dx.doi.org/10.1111/j.1600-0528.1991.tb00101.x

[7] Haugen, L.K. (1992) Palatine and mandibular tori. A morphologic study in the current Norwegian population. Acta Odontologica Scandinavica, 50, 65-77. http://dx.doi.org/10.3109/00016359209012748

[8] Kerdporn, D. and Sirirungrojying, S. (1999) A clinical study of oral tori in southern Thailand: Prevalence and the relation to parafunctional activity. European Journal of Oral Sciences, 107, 9-13. http://dx.doi.org/10.1046/j.0909-8836.1999.eos107103.x

[9] Sirirungrojying, S. and Kerdpon, D. (1999) Relationship between oral tori and temporomandibular disorders. International Dental Journal, 49, 101-104. http://dx.doi.org/10.1111/j.1875-595X.1999.tb00516.x

[10] Souza, E.A., Hotta, T.H. and Bataglion, C. (1996) Association of a temporomandibular disorder and Eagle's syndrome: Case report. Brazilian Dental Journal, 7, 53-58.

[11] Grossmann, E. and Paiano, G.A. (1998) Eagle's syndrome: A case report. The Journal of Craniomandibular Practice, 16, 126-130. http://dx.doi.org/10.5505/agri.2013.26779

[12] Ferreira de Albuquerque, R. Jr., Müller, K., Hotta, T.H. and Gonçalves, M. (2003) Temporomandibular disorder or Eagle's syndrome? A clinical report. Journal of Prosthetic Dentistry, 90, 317-320.

http://dx.doi.org/10.1016/S0022-3913(03)00522-5

[13] Shankland, W.E. (2010) Anterior throat pain syndromes: Causes for undiagnosed craniofacial pain. The Journal of Craniomandibular Practice, 28, 50-59.

[14] Fusco, D.J., Asteraki, S. and Spetzler, R.F. (2012) Eagle's syndrome: Embryology, anatomy, and clinical management. Acta Neurochirurgica, 154, 1119-1126. http://dx.doi.org/10.1007/s00701-012-1385-2

[15] Eagle, W.W. and Durham, N.C. (1949) Symptomatic elongated styloid process. Archives of Otolaryngology, 49, 490-503.

http://dx.doi.org/10.1001/archotol.1949.03760110046003
[16] Hotta, T.H., Vicente, M.F., dos Reis, A.C., Bezzon, O.L., Bataglion, C. and Bataglion, A. (2003) Combination therapies in the treatment of temporomandibular disorders: A clinical report. Journal of Prosthetic Dentistry, 89, 536-539.

[17] Leite, H.F., Niccoli Filho, W.D., Liberti, E.A., Madeira, M.C. and Simões, S. (1998) Prevalence of elongated stylohyoid ligament complex in human skulls. Revista Odontologica UNESP, 17, 145-151.

[18] Rizzatti-Barbosa, C.M., Ribeir, M.C., Silva-Concilio, L.R., Di Hipolito, O., Ambrosano GM. (2005) Is an elongated stylohyoid process prevalent in the elderly? A radiographic study in a Brazilian population. Gerodontology, 22, 112-115. http://dx.doi.org/10.1111/j.1741-2358.2005.00046.x

[19] Breault, M.R. (1986) Eagle's syndrome: Review of the literature and implications in craniomandibular disorders. The Journal of Craniomandibular Practice, 4, 323-337.

[20] Camarda, A.J., Deschamps, C. and Forest, D. (1989) Stylohyoid chain ossification: A discussion of etiology. Oral Surgery Oral Medicine Oral Pathology, 67, 508-514. http://dx.doi.org/10.1016/0030-4220(89)90264-8

[21] Krmpotić Nemanić, J., Vinter, I., Ehrenfreund, T. and Marusić, A. (2009) Postnatal changes in the styloid process, vagina processus styloidei, and stylomastoid foramen in relation to the function of muscles originating from the styloid process. Surgical and Radiologic Anatomy, 31, 343-348. http://dx.doi.org/10.1007/s00276-008-0450-2

[22] Helkimo, M. (1974) Studies on function and dysfunction of the masticatory system. II. Index for anamnestic and clinical dysfunction and occlusal state. Svensk Tandlakare Tidskrift, 67, 101-121.

[23] Hotta, P.T., Hotta, T.H., Bataglion, C., Bataglion, S.A., de Souza Coronatto, E.A., Siéssere, S. and Regalo, S.C.H. (2010) Emg analysis after laser acupuncture in patients with temporomandibular dysfunction (TMD)-Implications for practice. Complementary Therapies Clinical Practice, 16,158-160. http://dx.doi.org/10.1016/j.ctcp.2010.01.002

[24] Bajoria, A.A., Saldanha, S. and Shenoy, V.K. (2012) Evaluation of satisfaction with masticatory efficiency of new conventional complete dentures in edentulous patients-A survey. Gerodontology, 29, 231-238. http://dx.doi.org/10.1111/j.1741-2358.2012.00634.x

[25] de Felício, C.M., Mazzetto, M.O., de Silva, M.A., Bataglion, C. and Hotta, T.H. (2006) A preliminary protocol for multi-professional centers for the determination of signs and symptoms of temporomandibular disorders. The Journal of Craniomandibular Practice, 24, 258-264.

[26] Downie, W.W., Leatham, P.A., Rhind, V.M., Wright, V., Branco, J.A. and Anderson, J.A. (1978) Studies with pain rating scales. Annals of the Rheumatic Diseases, 37, 378381. http://dx.doi.org/10.1136/ard.37.4.378

[27] Hugoson, A., Bergendal, T., Ekfeldt, A. and Helkimo, M. (1998) Prevalence and severity of incisal and occlusal tooth wear in an adult Swedish population. Acta Odontologica Scandinavica, 46, 255-265.

http://dx.doi.org/10.3109/00016358809004775 
[28] Baba, K., Tsukiyama, Y., Yamazaki, M. and Clark, G.T. (2001) A review of temporomandibular disorder diagnostic techniques. Journal of Prosthetic Dentistry, 86, 184194. http://dx.doi.org/10.1067/mpr.2001.116231

[29] dos Reis, A.C., Hotta, T.H., Ferreira-Jeronymo, R.R., de Felício, C.M. and Ribeiro, R.F. (2000) Ear symptomatology and occlusal factors: A clinical report. Journal of Prosthetic Dentistry, 83, 21-24. http://dx.doi.org/10.1016/S0022-3913(00)70084-9

[30] Restrepo, C.C., Medina, I. and Patiño, I. (2011) Effect of Occlusal Splints on the Temporomandibular Disorders, Dental Wear and Anxiety of Bruxist Children. Eurean Journal of Dentistry, 5, 441-450.

[31] Jain, D., Chauhan, J.S, Jain, S. and Goel, G. (2011) Elongated styloid process: An unusual cause of neck pain and difficulty in swallowing. Journal of Orofacial Pain, 25, 269-271.
[32] Valerio, C.S., Peyneau, P.D., de Sousa, A.C., Cardoso, F.O., de Oliveira, D.R. and Taitson, P.F. (2012) Stylohyoid syndrome: Surgical approach. Journal of Craniofacial Surgery, 23, e138-140. http://dx.doi.org/10.1097/SCS.0b013e31824cdb46

[33] Borges Tde, F., Regalo, S.C., Taba, M. Jr., Siéssere, S., Mestriner, W. Jr. and Semprini, M. (2013) Changes in masticatory performance and quality of life in individuals with chronic periodontitis. Journal of Periodontology, 84, 325-331. http://dx.doi.org/10.1902/jop.2012.120069

[34] Bonjardim, L.R., Gavião, M.B., Pereira, L.J. and Castelo, P.M. (2005) Bite force determination in adolescents with and without temporomandibular dysfunction. Journal of Oral Rehabilitation, 32, 577-583. http://dx.doi.org/10.1111/j.1365-2842.2005.01465.x 